\title{
A Tractable Approximation of Chance Constrained Stochastic MPC based on Affine Disturbance Feedback
}

\author{
Frauke Oldewurtel, Colin N. Jones, Manfred Morari
}

\begin{abstract}
This paper deals with model predictive control of uncertain linear discrete-time systems with polytopic constraints on the input and chance constraints on the states. Recently, it has been shown that when having merely polytopic constraints and bounded disturbances, the conservatism of a robust solution can be reduced by applying a closed-loop prediction formulation. We show that in the presence of chance constraints and stochastic disturbances, this closed-loop formulation can be used together with a tractable approximation of the chance constraints to further increase the performance while giving probabilistic guarantees on the constraints.
\end{abstract}

\section{INTRODUCTION}

This paper deals with solving a model predictive control (MPC) problem for the class of discrete-time linear systems subject to stochastic disturbances. The aim is to provide a method for efficiently finding control policies that ensure to satisfy a given set of polytopic input constraints and uncertain linear constraints on the state, but which is sufficiently computationally tractable that it is also applicable to larger systems. The uncertain linear constraints are of the form

$$
\mathbf{P}(F x+G w \leq f) \geq 1-\alpha,
$$

where $x$ is the system state and $w$ is the disturbance. $f$ is a vector, $F$ and $G$ are matrices of appropriate sizes and $\alpha \in[0,1]$. This constraint requires that $F x+G w \leq f$ has to be fulfilled with probability $1-\alpha$. Such uncertain constraints that linearly depend on the disturbance as well as uncertain constraints of more complex structures are called chance constraints. If disturbances are to be accounted for in the formulation of an MPC problem, it is preferable to have future control inputs formulated as functions of future measured states. This is because in the future the disturbance realization and the system state will be known and, thus, the future predicted controller will have this information available while making a decision on the control action. This is usually called closed-loop prediction MPC.

\section{A. Closed-loop prediction MPC}

In so-called open-loop prediction MPC the control action that is predicted to be taken in the future is only a function of the current state, and not of future disturbance and state realizations. This is computationally very attractive. The disadvantage is that this very often results in highly conservative control behavior and infeasibility and instability problems can occur [10]. The reason for this is that a set of control actions over the entire horizon is chosen such

F. Oldewurtel, C. Jones and M. Morari are with the Department of Electrical Engineering, Automatic Control Laboratory, ETH Zurich, Switzerland \{oldewurtel, cjones, morari\}econtrol.ee.ethz.ch that the constraints are satisfied for all possible disturbance realizations. Thus the controller cannot respond to the disturbance and the prediction effectively assumes that the system will run in open-loop over the future horizon. In closed-loop prediction MPC the future control inputs are functions of future measured states and the decision variable in the resulting MPC optimization problem is exactly these functions. Optimizing over arbitrary functions is however in general not tractable. A popular approximation to this is "prestabilization", where a stabilizing linear state feedback is computed off-line and the online computation is restricted to a sequence of admissible offsets to the selected control law [2], [3], [11]. Since this can be quite conservative, an improvement to this approach would be to optimize over both the linear feedback control law and the offset sequence online. Unfortunately, this parametrization leads to a nonconvex set of feasible decision variables. There are recent results given in [1], [9], [17] that describe one approach to addressing this problem. The authors propose to have the control policy parameterized as an affine function of the disturbances, which leads to a convex set of feasible decision variables. This affine disturbance feedback parametrization is shown to be equivalent to the affine state feedback parametrization in [7] in the sense that is leads to the same control inputs. In [1], [9] and [7] bounded disturbances are assumed, whereas in [17] stochastic disturbances are considered. Unfortunately, with the method in [17] the problem that was originally an LP is turned into a second order cone problem. Consequently, it is not applicable to large-scale problems, which are our primary interest here.

\section{B. Chance constraints}

An example of a control problem, which naturally leads to a chance constraint of the form given in (1) and which is the primary motivation for the work, originates from building climate control. The European standards state that the room temperature must be kept within a certain range with a certain probability. The control problem is then to satisfy this chance constraint while using a minimum amount of energy. Problems of uncertain linear systems with chance constraints are very common and can also be found for example in finance, physics, aeronautics etc. [14].

Chance constraints are in general hard to deal with and many approximations exist. The interested reader is referred to for example [4], [13], [15]. In [4] the authors suggest to assume that the uncertainty is bounded by a certain value, which effectively approximates the chance constraint with a hard constraint. The authors describe how the bounding 
value has to be chosen such that they can give a performance guarantee on each chance constraint. The idea of bounding the disturbance and approximating the chance constraint with a hard constraint fits very well into the MPC framework since it leads to a classic robust control problem. Furthermore, this approximation has the important advantage that it preserves the original structure of the problem, so for example an LP stays an LP. In [5] the authors apply the approximation of [4] in open-loop prediction MPC. We suggest in this paper to extend it to closed-loop prediction MPC.

\section{Main Idea and Outline}

The main idea of this paper is to use the approximation of the chance constraint in [4] and combine it with the affine disturbance feedback formulation in [1], [17], [9]. This enables us to formulate the MPC problem as a conservative classic robust control problem. Our aim is to reduce the conservativeness of the control by applying closed-loop prediction MPC and further by making use of the fact that the chance constraints may be violated from time to time. The fact that the proposed formulation preserves the original structure makes it applicable to large-scale systems as used for example in building climate control.

Section 2 states the class of system that is to be considered throughout the paper and lists a number of standing assumptions. Section 3 is divided in three parts. The first part describes the affine disturbance feedback parametrization. The second part describes the approximation of chance constrained problems. The third part introduces the new methodology that combines the affine disturbance feedback and the chance constraint approximation. In Section 4 simulation results are presented. Section 5 concludes with a discussion and an outlook.

\section{PRELIMINARIES}

Consider the following stable discrete-time LTI system

$$
x^{+}=A x+B u+E w,
$$

where $x \in \mathbb{R}^{n}$ is the system state at the current time instant, $x^{+}$is the state at the next time instant, $u \in \mathbb{R}^{m}$ is the control input and $w \in \mathbb{R}^{p}$ is a stochastic disturbance. The disturbances are assumed to be independent and identically normal distributed random variables $(w \sim \mathcal{N}(0, I))$. The system is subject to input constraints

$$
\mathcal{U}:=\left\{u \in \mathbb{R}^{m} \mid S u \leq s\right\},
$$

where $S \in \mathbb{R}^{q \times m}$ and $s \in \mathbb{R}^{q}$ and $\mathcal{U} \subset \mathbb{R}^{m}$ is a bounded, polytopic set. There are also polytopic constraints on the state

$$
\mathcal{X}:=\left\{x \in \mathbb{R}^{n} \mid F x \leq f\right\},
$$

where $F \in \mathbb{R}^{r \times n}, f \in \mathbb{R}^{r}$ and $\mathcal{X} \subset \mathbb{R}^{n}$. The state constraints are given as chance constraints, i.e.

$$
\mathbf{P}(x \in \mathcal{X}) \geq 1-\alpha .
$$

Define $\mathbb{Z}_{[k, l]}$ as the set of integers $\{k, k+1, \ldots, l\}$.

\section{METHOD}

Let $N$ be the length of the planning horizon of the control problem and define

$$
\mathbf{w}:=\left[w_{0}^{T} \ldots w_{N-1}^{T}\right]^{T},
$$

where $\mathbf{w} \in \mathbb{R}^{m \cdot N}$ denotes a disturbance sequence over the interval 0 to $N-1$. In order to have an effective control in the presence of uncertainties, we suggest to apply an affine disturbance feedback, so the control policy $\mu$ is defined by

$$
\mu:=\left\{\mu_{0}, \mu_{1}(\cdot), \ldots, \mu_{N-1}(\cdot)\right\},
$$

where $\mu_{0} \in \mathcal{U}$ and $\mu_{i}: \mathbb{R}^{p} \rightarrow \mathcal{U}, i=1, \ldots, N-1$ is a mapping from the disturbance realization to the set of inputs. $\mu_{0}$ is a control action since the current state is known, whereas each $\mu_{k}(\cdot)$ is a disturbance feedback control law. Let $\phi_{i}\left(x_{0}, \mu, \mathbf{w}\right)$ denote the solution to (2) at time $i$ when the state is $x_{0}$ at time 0 , the disturbance realization is $w_{0}, \ldots, w_{i-1}$, and the control law $\mu_{j}$ is applied at time $j$. Then the chance constraints can be written as

$$
\mathcal{X}_{c}=\left\{\mathbf{P}\left(\phi\left(x_{0}, \mu, \mathbf{w}\right) \in \mathcal{X}\right) \geq 1-\alpha\right\},
$$

where $\mathcal{X}_{c} \subset \mathbb{R}^{n}$. Now we can state the MPC problem that we would like to solve. We define an optimal policy to be one that minimizes the value of a cost function that is linear in the disturbance free state and input sequences.

\section{Definition 1 (MPC problem):}

$$
\begin{aligned}
J\left(x_{0}\right):= & \min _{\mu} \sum_{i=0}^{N-1}\left\|Q \phi_{i}\left(x_{0}, \mu, \mathbf{0}\right)\right\|_{p}+\left\|R \mu_{i}\left(x_{0}, \mathbf{0}\right)\right\|_{p} \\
\text { s.t. } & S \mu_{i}\left(x_{0}, \mathbf{w}\right) \leq s, \quad \forall i \in \mathbb{Z}_{[0, N-1]} \\
& \mathbf{P}\left(F \phi_{i}\left(x_{0}, \mu, \mathbf{w}\right) \leq f\right) \geq 1-\alpha \forall i \in \mathbb{Z}_{[1, N]} \\
& \mathbf{w} \sim \mathcal{N}(0, I),
\end{aligned}
$$

where $\|\cdot\|_{p}$ can be any polytopic norm. The problem in (9) cannot be solved for the following reasons

- We cannot optimize over arbitrary functions.

- A chance constraint as in (9) is non-convex and generally intractable [4].

In order to tackle the above mentioned problems we propose to find approximations. The first approximation defines an appropriate structure for the control policies that enables us to formulate a tractable optimization problem. The second approximation replaces the chance constraints with conservative simple linear bounds while guaranteeing the satisfaction of the chance constraint and disposing of the stochastic description of the disturbances. The proposed approximations are introduced in the next two sections.

\section{A. Affine Disturbance Feedback}

Consider a simplified version of the problem in (9): 
Definition 2 (Simplified MPC problem):

$$
\begin{aligned}
J_{s p}\left(x_{0}\right)= & \min _{\mu} \sum_{i=0}^{N-1}\left\|Q \phi_{i}\left(x_{0}, \mu, \mathbf{0}\right)\right\|_{p}+\left\|R \mu_{i}\left(x_{0}, \mathbf{0}\right)\right\|_{p} \\
\text { s.t. } & \left.S \mu_{i}\left(x_{0}, \mathbf{w}\right)\right) \leq s, \quad \forall i \in \mathbb{Z}_{[0, N-1]} \\
& F \phi_{i}\left(x_{0}, \mu, \mathbf{w}\right) \leq f, \quad \forall i \in \mathbb{Z}_{[1, N]} \\
& \forall \mathbf{w} \in \mathcal{B}^{N},
\end{aligned}
$$

where $\mathcal{B}:=\{w \mid\|w\| \leq b\}$ and $\mathcal{B}^{N}:=\mathcal{B} \times \ldots \times \mathcal{B}$. This is the same problem as in (9), but with the disturbance w bounded in the set $\mathcal{B}^{N}$ and the chance constraint replaced by a hard constraint.

Recently, a tractable formulation to solve robust optimization problems with hard constraints, the so called adjustable robust counterpart, was presented in [1], [17], [9]. The authors propose to have the control policy parameterized as an affine function of past disturbances, which leads to a convex set of feasible decision variables.

Definition 3 (Affine disturbance feedback):

$$
\mu_{i}\left(x_{0}, \mathbf{w}\right):=\sum_{j=0}^{i-1} M_{i, j} w_{j}+h_{i},
$$

with $M_{i, j} \in \mathbb{R}^{m \times p}$ and $h_{i} \in \mathbb{R}^{m}$. Define

$$
\begin{gathered}
\pi:=\left[\begin{array}{llll}
\mu_{0}^{T} & \mu_{1}(\cdot)^{T} & \ldots & \mu_{N-1}^{T}(\cdot)
\end{array}\right]^{T} \text { and } \\
\mathbf{M}:=\left[\begin{array}{cccc}
0 & \ldots & \ldots & 0 \\
M_{1,0} & 0 & \ddots & 0 \\
\vdots & \ddots & \ddots & \vdots \\
M_{N-1,0} & \cdots & M_{N-1, N-2} & 0
\end{array}\right], \mathbf{h}:=\left[\begin{array}{c}
h_{0} \\
\vdots \\
\vdots \\
h_{N-1}
\end{array}\right] .
\end{gathered}
$$

Then the inputs can be written as $\pi=\mathbf{M w}+\mathbf{h}$.

Remark 1: Note that with the definitions in (7) and (12), $\mu_{i}$ clearly equals the $m \cdot i+1$-th up to the $(i+1) \cdot m$-th row of $\pi$.

Theorem 4: [7]

Let the set $\mathcal{B}$ from (10) be a polytope and the receeding horizon control law $\mu_{i}(\cdot)$ be defined as in (11). Then the origin can be shown to be input-to-state stable for the closed-loop system under the additional standard requirement that the final state $x_{N}$ is constrained to lie in an appropriately defined terminal set and the cost includes a standard final weighting term $V_{N}\left(x_{N}\right)$.

Remark 2: Throughout the paper we will assume for brevity the inclusion of appropriate terminal sets and weights such that (10) generates a stabilizing control law when applied in a receding horizon fashion. See [7] for details.

To summarize, the affine disturbance feedback parametrization in (11) gives us a tractable approximation for the problem in (10). However, this closed-loop prediction formulation is dealing with bounded disturbances and does not contain chance constraints. In order to tackle the problem in (9) we will next look at approximations of chance constraints that can be used with the affine disturbance formulation.

\section{B. Approximation of chance constraints}

In this section we will first investigate the structure of the chance constraint, reformulate it and then use the approximation in [4].

Chance constraint problems are a well studied topic in optimization theory. The chance constraint of the MPC problem in (9) can be rewritten as

$$
\begin{aligned}
& \mathbf{P}\left(F \phi_{i}\left(x_{0}, \mu, \mathbf{w}\right)-f \leq 0\right) \\
& =\int_{\mathbf{w} \in\left\{\mathbf{w} \mid F \phi_{i}\left(x_{0}, \mu, \mathbf{w}\right)-f \leq 0\right\}} p d f(\mathbf{w}) \mathrm{d} \mathbf{w} \geq 1-\alpha
\end{aligned}
$$

where $p d f$ is the probability density function of w. In general, one distinguishes between individual and joint chance constraints. Given a set of $n$ chance constraints, individual chance constraints mean that each chance constraint has to be fulfilled with the given probability whereas joint chance constraints mean that all of the $n$ constraints have to be fulfilled with the given probability [6]. In (13) the chance constraint has to be satisfied for each time step $i$ in the future individually, however, since at each time step there are $r$ inequalities that have to be fulfilled, we have a joint chance constraint at each time step. Since individual chance constraints are far easier to handle, the usual procedure is to approximate the joint chance constraints with individual chance constraints and set the probability level by defining $\alpha_{k}=\alpha / r$, where $r$ is the number of rows [12]. This gives

$$
\begin{aligned}
& \mathbf{P}\left(F_{k} \phi_{i}\left(x_{0}, \mu, \mathbf{w}\right)-f_{k} \leq 0\right) \geq 1-\alpha_{k}=1-\alpha / r \\
& k \in \mathbb{Z}_{[1, r]}
\end{aligned}
$$

where $F_{k}$ and $f_{k}$ denote the $k$-th row of $F$ and $f$ respectively. When using the affine disturbance parametrization in (11) and defining

$$
\Phi:=\left[\phi_{1}^{T}(\cdot), \ldots, \phi_{N}^{T}(\cdot)\right]^{T},
$$

we can write

$$
\Phi=\mathbf{A} x_{0}+\mathbf{H h}+\mathbf{L M w}+\mathbf{E w},
$$

and the chance constraints on the future states can then be written (by omitting the probability) as

$$
\mathbf{F}\left(\mathbf{A} x_{0}+\mathbf{H h}+\mathbf{L M w}+\mathbf{E w}\right)-\mathbf{f},
$$

where the matrices $\mathbf{F}, \mathbf{A}, \mathbf{H}, \mathbf{L}, \mathbf{E}$ and $\mathbf{f}$ are derived from the system equation and constraint matrices and can be found in the appendix. The constraints on the states in the first time step are represented by the first $r$ rows. The constraints on the state in the second time step in the next $r$ rows and so on. Each row of (17) can be written as

$$
\begin{aligned}
& \mathbf{F}_{k}\left(\mathbf{A} x_{0}+\mathbf{H h}+\mathbf{L M w}+\mathbf{E w}\right)-\mathbf{f}_{\mathbf{k}} \leq 0 \\
& k \in \mathbb{Z}_{[1, r \cdot N]},
\end{aligned}
$$


which we write in the simpler form

$$
\left(a^{0}+\Delta A \mathbf{w}\right)^{T} \mathbf{z}-\left(b^{0}+\Delta b \mathbf{w}\right)^{T} \leq 0,
$$

where $a^{0}$ is a vector of length $m \cdot N \cdot p \cdot N, \Delta A$ is a matrix of size $m \cdot N \cdot p \cdot N \times p \cdot N, b^{0}$ is a scalar, $\Delta b$ is a vector of length $p \cdot N$ and $\mathbf{z}$ is a vector of length $m \cdot N \cdot p \cdot N+m \cdot N$ containing the decision variables $\mathbf{M}$ and $\mathbf{h}$ in vectorized form. The only difficulty in the derivation of the equivalence of (18) and (19) is to get $\mathbf{M}$ on the right hand side in $\mathbf{F}_{k} \mathbf{L M w}$. The derivation of that can be found in the appendix. In [4] the authors propose to bound the disturbance such that we have a new disturbance $v \in \mathbb{R}^{p}$. We can then write the resulting uncertainty set as

$$
\mathcal{U}_{\Omega}=\left\{\left[\begin{array}{l}
a^{0} \\
b^{0}
\end{array}\right]+\left[\begin{array}{c}
\Delta A \\
\Delta b
\end{array}\right] \mathbf{v},\|v\| \leq \Omega\right\} .
$$

Thus, the chance constraint of the form (19) can be approximated with a hard constraints of the form

$$
\max _{\|v\| \leq \Omega}\left(a^{0}+\Delta A \mathbf{v}\right)^{T} \mathbf{z}+\left(b^{0}+\Delta b \mathbf{v}\right) \leq 0
$$

The authors in [4] proved the following very useful theorem.

Theorem 5: [4]

With the model of uncertainty in (20) and under the assumption that $\mathbf{w} \sim \mathcal{N}(0, I)$, we have the probability bound:

$$
\begin{aligned}
& \mathbf{P}\left(\left(a^{0}+\Delta A \mathbf{w}\right)^{T} \mathbf{z}-\left(b^{0}+\Delta b \mathbf{w}\right)^{T}>0\right) \\
& \quad \leq \sqrt{e} \cdot \Omega \cdot \exp \left(-\frac{\Omega^{2}}{2}\right) .
\end{aligned}
$$

This theorem gives us a performance guarantee in the following sense. If we choose $\Omega$ according to Theorem 5 and bound the disturbance $\mathbf{w}$ as per (20), then it is guaranteed that the constraint in (14) is fulfilled.

As a result, and this is the main idea of this paper, we can use this approximation and solve a conservative classic robust control problem. This is shown in the next section.

\section{Closed loop prediction with chance constraints}

With the combination of the two approximations, the affine disturbance feedback parametrization and the approximation of the chance constraint, we can now state a tractable approximation of the MPC problem in (9).

$$
\begin{gathered}
J_{c c}\left(x_{0}\right)=\min _{\mathbf{M}, \mathbf{h}} \sum_{i=0}^{N-1}\left\|\left[Q \mathbf{A} x_{0}+Q \mathbf{H h}\right]_{i \cdot n+1:(i+1) \cdot n}\right\|_{p} \\
+\left\|R h_{i}\right\|_{p} \\
\text { s.t. } \max _{\|v\| \leq \Omega} \mathbf{S M v}+\mathbf{S h} \leq \mathbf{s} \\
\max _{\|v\| \leq \Omega} \mathbf{F}\left(\mathbf{A} x_{0}+\mathbf{H h}+\mathbf{L M v}+\mathbf{E v}\right)-\mathbf{f} \leq 0,
\end{gathered}
$$

where $\mathbf{S}:=I \otimes S$ and $\otimes$ is the Kronecker product. The constraints involving maximization in (23) involve products of the disturbance $\mathrm{v}$ and the decision variable $\mathrm{M}$. We follow the approach made standard in robust programming and take the duals of these linear optimization problems. This dualization is shown for one example. Each constraint has to be considered row-wise. We have for example

$$
\begin{aligned}
& \max \mathbf{F}_{k} \mathbf{A} x_{0}+\mathbf{F}_{k} \mathbf{H h}-\mathbf{f}_{k}+\left(\mathbf{G}^{\mathbf{k}} \mathbf{m}+\mathbf{E}_{k}\right)^{T} \mathbf{v} \leq 0 \\
& \text { s.t. }-\Omega \mathbf{1} \leq \mathbf{v} \leq \Omega \mathbf{1},
\end{aligned}
$$

where $\mathbf{G}^{k}$ is a matrix resulting from rearranging $\mathbf{F}_{k} \mathbf{L M w}$ and can be found in the appendix. The dual of this optimization problem is

$$
\begin{array}{ll}
\min & \Omega\left(\mathbf{1}^{T} \lambda_{l}^{k}+\mathbf{1}^{T} \lambda_{u}^{k}\right)+\mathbf{F}_{k} \mathbf{A} x_{0}+\mathbf{F}_{k} \mathbf{H h}-\mathbf{f}_{k} \geq 0 \\
\text { s.t. } & \lambda_{l}^{k}-\lambda_{u}^{k}=\mathbf{G m}+\mathbf{E}_{k} \\
& \lambda_{l}^{j}, \quad \lambda_{u}^{j} \geq 0, \quad \forall k \in \mathbb{Z}_{[1, r \cdot N]}
\end{array}
$$

By duality, we have that any feasible $\lambda$ in (25) will upper bound the maximization in (24). We are therefore free to drop the minimization in the constraint of (25) and by strong duality on linear programming, this relaxation will be tight. This results in the following linear program.

\section{Definition 6: Closed loop prediction MPC with chance constraints}

$$
\begin{aligned}
& J_{c d}\left(x_{0}\right)=\min _{\mathbf{M}, \mathbf{h}} \sum_{i=0}^{N-1}\left\|Q\left[\mathbf{A} x_{0}+\mathbf{H h}\right]_{i \cdot n+1}:(i+1) \cdot n\right\|_{p} \\
& +\left\|R h_{i}\right\|_{p} \\
& \text { s.t. } \Omega\left(\mathbf{1}^{T} \kappa_{l}^{j}+\mathbf{1}^{T} \kappa_{u}^{j}\right)+\mathbf{S}_{j} \mathbf{h}-\mathbf{s}_{j} \geq 0 \\
& \kappa_{l}^{j}-\kappa_{u}^{j}=\left(\mathbf{S}_{j} \mathbf{M}\right)^{T} \\
& \kappa_{l}^{j}, \kappa_{u}^{j} \geq 0 \quad \forall j \in \mathbb{Z}_{[1, q \cdot N]} \\
& \Omega\left(\mathbf{1}^{T} \lambda_{l}^{k}+\mathbf{1}^{T} \lambda_{u}^{k}\right)+\mathbf{F}_{k} \mathbf{A} x_{0}+\mathbf{F}_{k} \mathbf{H h}-\mathbf{f}_{k} \geq 0 \\
& \lambda_{l}^{k}-\lambda_{u}^{k}=\mathbf{G}^{k} \mathbf{m}+\mathbf{E}_{k} \\
& \lambda_{l}^{k}, \lambda_{u}^{k} \geq 0, \quad \forall k \in \mathbb{Z}_{[1, r \cdot N]}
\end{aligned}
$$

We know however, that $\|v\| \leq \Omega$ is just an artificial bound on the disturbance $w$ and the actual disturbance is normally distributed $w \sim \mathcal{N}(0, I)$. It is therefore possible for $\|w\|$ to be larger than $\Omega$ by a sufficient amount that it drives the initial state $x_{0}$ outside of the feasible set of (26).

Even if $\|w\|>\Omega$ it can happen that we still remain in the feasible set of the constraints. However, if we do not and the controller in (26) does not provide a feasible solution, then we have to apply a backup controller.

In the case that we are driven outside the feasible set, we suggest to soften the constraints and to solve the open-loop problem, which will act to drive the state back to the feasible set of (26). It is reasonable to soften the state constraints when necessary because, being chance constraints, we are free to violate them on occasion (although in this case, the chance constraints will temporarily not be met).

\section{RESULTS}

\section{A. Simulations}

To test the proposed methods we look at a small building example in the form of (2). The idea here is to take weather and occupancy forecasts into account in the control. The system has three states. Let $x_{1}$ be the room temperature, 
$x_{2}$ the temperature in the wall connected with another room and $x_{3}$ the temperature in the wall connected to the outside. The system is subject to disturbances $w_{1}$ being the outside temperature, $w_{2}$ being the solar radiation and $w_{3}$ being internal heat gains like people, computers etc. There is a forecast of these disturbances available that is subject to uncertainty. Consequently, the disturbance realization is the predicted disturbance plus a random value. The control objective is to keep the room temperature above $21^{\circ} \mathrm{C}$ with minimum energy. The single available constrained input $u_{1}$ is the heating. The system matrices are a simplified version of the building example investigated in [8] and are given as

$$
\begin{aligned}
& A=\left[\begin{array}{lll}
0.8511 & 0.0541 & 0.0707 \\
0.1293 & 0.8635 & 0.0055 \\
0.0989 & 0.0032 & 0.7541
\end{array}\right], \quad B=\left[\begin{array}{l}
0.0035 \\
0.0003 \\
0.0002
\end{array}\right] \\
& E=\left[\begin{array}{ccc}
22.2170 & 0.0018 & 0.0035 \\
1.5376 & 0.0007 & 0.0003 \\
103.1813 & 0.0001 & 0.0002
\end{array}\right] \quad C=\left[\begin{array}{lll}
1 & 0 & 0
\end{array}\right]
\end{aligned}
$$

Please note that this is a highly simplified version of a building presented here for illustrative purposes. Future investigations will examine the applicability of the proposed approach in more realistic scenarios. An extraction of the predicted disturbances can be found in Figure 1.

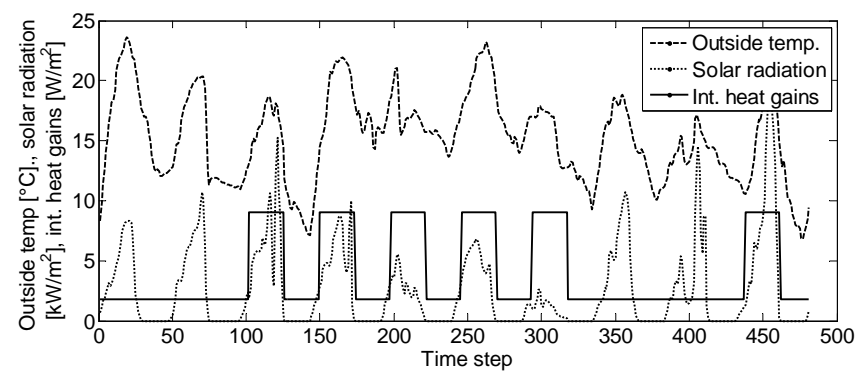

Fig. 1. Outside temperature, solar radiations and internal heat gains. The outside temperature fluctuates within the day. The internal heat gains consist of a constant part and a part that is changing with the daytime. This is because an office building is assumed with people being absent during the night and on weekends. Solar radiation is equal to 0 at night.

We compared the behavior of two different controllers, an open-loop prediction MPC and a closed-loop prediction MPC based on affine disturbance feedback as suggested in this paper. Both controllers are subject to stochastic disturbances but assume a bound on the disturbance. The magnitude of this bound is changed in order to investigate the effect on the energy consumption. In all simulations the system is subject to the same disturbance realizations. Figure 2 shows the an extraction for open-loop prediction MPC with probability of constraint violation $0.5 \%$ (OLP0.5), of closedloop prediction MPC with probability of constraint violation $0.5 \%$ (CLP0.5), and of closed-loop MPC with probability of constraint violation 5\% (CLP5.0). For OLP0.5 and CLP0.5 the room temperature is often high above $21^{\circ} \mathrm{C}$. However CLP0.5 ensures that the room temperature is not as much over $21^{\circ} \mathrm{C}$ as for OLP0.5, so it leads to a less conservative control behavior. CLP5.0 leads to a room temperature that is

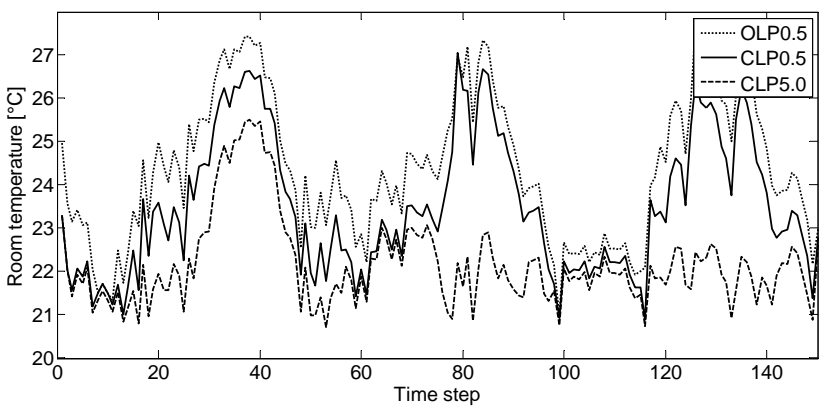

Fig. 2. Controller comparison. Open-loop control leads to a very conservative control behavior. Room temperature is often high above $21^{\circ} \mathrm{C}$. Closed-loop prediction MPC is less conservative. If we put a smaller bound on the disturbance, the closed-loop prediction MPC the conservativeness is significantly further decreased at the expense of slightly being below $21 \mathrm{C}$ from time to time.

often just very little over $21^{\circ} \mathrm{C}$ at the expense of violating the $21^{\circ} \mathrm{C}$ more often than the other two controllers. Numerical results of the simulations can be found in Table I.

TABLE I

Overview of controller performances.

\begin{tabular}{lcc}
\hline Controller & Energy usage $\left[\mathrm{kW} / \mathrm{m}^{2}\right]$ & Constraint violation [\%] \\
\hline OLP MPC & 401.375 & 0.1 \\
CLP MPC & 353.704 & 0.1 \\
CLP MPC & 326.542 & 0.7 \\
CLP MPC & 267.097 & 6.5 \\
\hline
\end{tabular}

It can be seen from Table I that there is a tradeoff between having a low energy consumption and a high degree of constraint satisfaction. This tradeoff-curve is depicted in Figure 3.

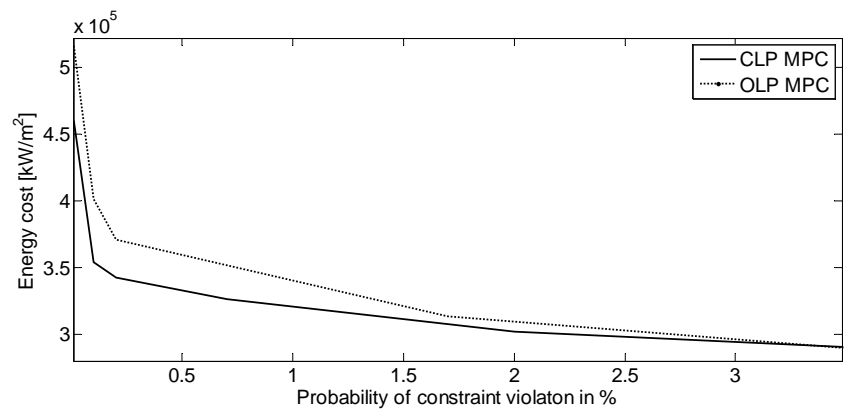

Fig. 3. Tradeoff curve for energy consumption and constraint violation. The curve depicts the tradeoff between a low energy consumption and a high degree of constraint satisfaction.

From the tradeoff-curve in Figure 3 we see that the energy consumption can be reduced allowing more constraint violation. Decreasing the degree of constraint satisfaction just by a small amount leads to a large effect in the energy consumption. If the degree of constraint satisfaction is further decreased this effect saturates. 


\section{B. Discussion}

We have seen that in the above example the conservativeness of the control behavior in open-loop prediction MPC can already be significantly reduced by closed-loop prediction MPC, which leads to a lower energy consumption. By taking into account the chance constraint formulation the energy consumption can further be significantly reduced. Taking into account the chance constraint formulation consequently leads to more constraint violations. However, for some applications as for example the chosen one from building climate control, this violation from time to time is defined in the standards and perfectly valid. So making use of the chance constraint formulation and applying closed-loop prediction MPC has significantly improved the controller performance.

\section{CONCLUSIONS AND FUTURE WORK}

\section{A. Conclusions}

We have shown a new method for solving a model predictive control (MPC) problem for the class of discretetime linear systems subject to polytopic input constraints and chance constraints on the states in the presence of stochastic disturbances. This method combines an approximation for closed-loop prediction MPC, the affine disturbance feedback, and an approximation of the chance constraints. We have shown that by using this method the conservativeness of a solution can be significantly reduced. This improvement is due on the one hand to the closed-loop prediction formulation and on the other to the flexibility given by the chance constraints.

\section{B. Future Work}

Having said that the primary motivation for this work is an application in building climate control one future goal certainly is to do testing with more realistic models and then field tests on the Siemens building control testbed.

\section{ACKNOWLEDGMENTS}

The authors gratefully acknowledge the contribution of Siemens Building Technologies, the Building Technologies Laboratory of EMPA Dübendorf and the Federal Institute for Meterology and Climatology MeteoSwiss.

\section{APPENDIX}

$$
\begin{gathered}
\mathbf{A}:=\left[\begin{array}{c}
A \\
A^{2} \\
A^{3} \\
\vdots \\
A^{N}
\end{array}\right] \quad \mathbf{H}:=\left[\begin{array}{ccccc}
B & 0 & \ldots & \ldots & 0 \\
A B & B & 0 & \ldots & 0 \\
A^{2} B & A B & B & 0 & \ldots \\
\vdots & \vdots & \vdots & \vdots & \ddots
\end{array}\right] \\
\mathbf{L}:=\left[\begin{array}{cccc}
0 & \ldots \\
B M_{1,0} & 0 & \ldots & 0 \\
A B M_{1,0}+B M_{2,0} & B M_{2,1} & 0 & \ldots \\
\vdots & \vdots & \vdots & \ddots
\end{array}\right] \quad \mathbf{E}:=\left[\begin{array}{cccc}
E & 0 & \ldots & 0 \\
A E & E & 0 \\
A^{2} E & A E & E & \ldots \\
\vdots & \vdots & \vdots & \ddots
\end{array}\right] \\
\mathbf{F}=I \otimes F \quad \mathbf{f}:=\left[f^{T} \ldots f^{T}\right]^{T}
\end{gathered}
$$

$\mathbf{F}_{k} \mathbf{L M w}=g^{T} \mathbf{M w}$, where $g^{T}$ is the vector resulting from $\mathbf{F}_{k} \mathbf{L}$. Since $g^{T} \mathbf{M w}$ is scalar, we just need to show that $g^{T} \mathbf{M w}=\mathbf{m G}$ for appropriate vectorized $\mathbf{m}$ and $\mathbf{G}$, since we can just take the transpose. We have that $g_{k} M_{i, j}=$ $\left[m_{* 0}^{T} \ldots m_{* p}^{T}\right]^{T}\left(I \otimes g_{k}\right)$, where $m_{* j}$ is the $j$-th column of $M_{i, j}$. Then the multiplication of each column of $\mathbf{M}$ is

$$
M_{* j}^{T}\left[\begin{array}{c}
I \otimes g_{0} \\
I \otimes g_{1} \\
\vdots
\end{array}\right]=M_{* j}^{T} \hat{G}
$$

Then we can write $\left[M_{* 0}^{T} \ldots M_{N}^{T}\right]^{T}(I \otimes \hat{G})=:$ mG.

\section{REFERENCES}

[1] A. Ben-Tal, A. Goryashko, E. Guslitzer, A. Nemirovski, "Adjustable robust solutions of uncertain linear programs", Math. Program., vol. 99(2), 2004, pp. 351-376.

[2] A. Bemporad, "Reducing conservatism in predictive control of constrained systems with disturbances", Proc. IEEE Conference on Decision and Control, vol. 37, 1998, pp. 1384-1389.

[3] A. Bemporad, M. Morari, "Robust model predictive control: A survey", Robustness in identification and control, Ed: Garulli, A., Tesi, A., Vicino, A., 1999, pp. 207-226.

[4] D. Bertsimas, M. Sim, "Tractable Approximations to Robust Conic Optimization Problems", Math. Program., ser. B 107, 2006, pp. 5-36.

[5] D. Bertsimas, D. B. Brown, "Constrained Stochastic LQC: A tractable approach", IEEE Transactions on automatic control, vol. 52(10), 2007, pp. 1826-1841.

[6] W. Chen, M. Sim, J. Sun, C.-P. Teo, "From CVaR to Uncertainty Set: Implications to Joint Chance Constrained Optimization", Working paper, NUS Business School, 2007

[7] P. J. Goulart, E. C. Kerrigan, J. M. Maciejowski, "Optimization over state feedback policies for robust control with constraints", Automatica, vol. 42, 2006, pp. 523-533.

[8] M. Gwerder, J. Toedtli, "Predictive control for integrated room automation", CLIMA 2005, Lausanne, 2005.

[9] J. Löfberg, "Approximations of closed-loop MPC", Proc. of 42nd Conf. on Decision and Control, 2003, pp. 1438-1442.

[10] D. Q. Mayne, J. B. Rawlings, C. V. Rao, P. O. M. Skokaert, "Constrained model predictive control: Stability and optimality", $\mathrm{Au}$ tomatica, vol. 36(6), 2000, pp. 789-814.

[11] D. Q. Mayne, M. M. Seron, S. V. Rakovic, "Robust model predictive control of constrained linear systems with bounded disturbances", Automatica, vol. 41(2), 2005, pp. 219-224.

[12] L. B. Miller, H. Wagner, "Chance-constrained programming with joint constraints", Oper. Res., vol. 13, 1965, pp. 930-945.

[13] A. Nemirovski, A. Shapiro, "Convex approximations of chance constrained programs", Siam J. Optim., vol. 17(4), pp. 969-996.

[14] B. Pasik-Duncan, R. Elliott, M. Davis, "Guest Editorial, Special Issue on Stochastic Control", IEEE Trans. on Automatic Control, vol. 49(3), 2004, pp. 321-323.

[15] A. Prékopa, "Stochastic Programming", Kluwer Academic Publishers, Dordrecht, The Netherlands.

[16] P. O. M. Skokaert, D. Q. Mayne, "Min-max feedback model predictive control for constrained linear systems", IEEE Trans. Autom. Control, vol. 43(8), 1998, pp. 1136-1142.

[17] D. H. van Hessem, O. H. Bosgra, "A conic reformulation of model predictive control including bounded and stochastic disturbances under state and input constraints", Proc. of 41st IEEE Conf. on Decision and Control, 2002, pp. 4643-4648 